International Journal of Wireless \& Mobile Networks (IJWMN) Vol. 4, No. 4, August 2012

\title{
The Landscape of Wireless Sensing in Greenhouse Monitoring and Control
}

\author{
Krzysztof S. Berezowski ${ }^{1,2}$ \\ Institute of Computer Engineering, Control and Robotics (CECR); \\ ${ }^{1}$ Wrocław University of Technology; Wybrzeże Wyspiańskiego 27; 50-370 Wrocław; \\ Poland \\ ${ }^{2}$ Research \& Engineering Center, Ltd.; Strzegomska 46B, 53-611 Wrocław, Poland
}

\begin{abstract}
In this paper we rethink the application of short-range radio communication technologies to greenhouse management. We briefly survey the application domain, the technological landscape and directions; then review the most interesting properties of existing deployments, and finally define some possible research ideas in the field. This paper neither assumes nor surveys certain WSN technologies, rather than that it attempts to define research problems, identify design space constraints and sketch some possible solutions. The purpose of this paper is twofold: to make computer engineer more aware about this specific application domain and the space it offers for applying IT and communication infrastructure, as well as to make horticulture researchers more aware of what wireless technologies have to offer and how to optimize their usage in the greenhouse.
\end{abstract}

Keywords: greenhouse, climate control, water management, wireless sensor networks, WSN, radio-frequency identification, RFID

\section{Introduction}

While Google Scholar returns an approx. number of 83700 hits1 of the keyword intitle: greenhouse, and more than 500000 hits of the keyword "wireless sensor network", only 383 hits sustain an attempt to create the inner product of the two. A slightly more accurate search2 returns 210 documents mentioning wireless networking in general or ZigBee, its most popular incarnation, in the context of greenhouse applications directly in the title. Within this set, nearly no papers were published before 2005, and the number of papers published yearly since 2005 (Figure 1) clearly shows that interest in applying wireless networking in greenhouse monitoring and control, although relatively recent, has quickly been gaining momentum.

1 All queries mentioned in the introduction were run in June, 2012.

2 (intitle:horticulture OR intitle:greenhouse OR intitle:"green house" OR intitle:"green-

house") AND (intitle:zigbee OR intitle:WSN OR intitle:wireless)

DOI : 10.5121/ijwmn.2012.4410 
International Journal of Wireless \& Mobile Networks (IJWMN) Vol. 4, No. 4, August 2012

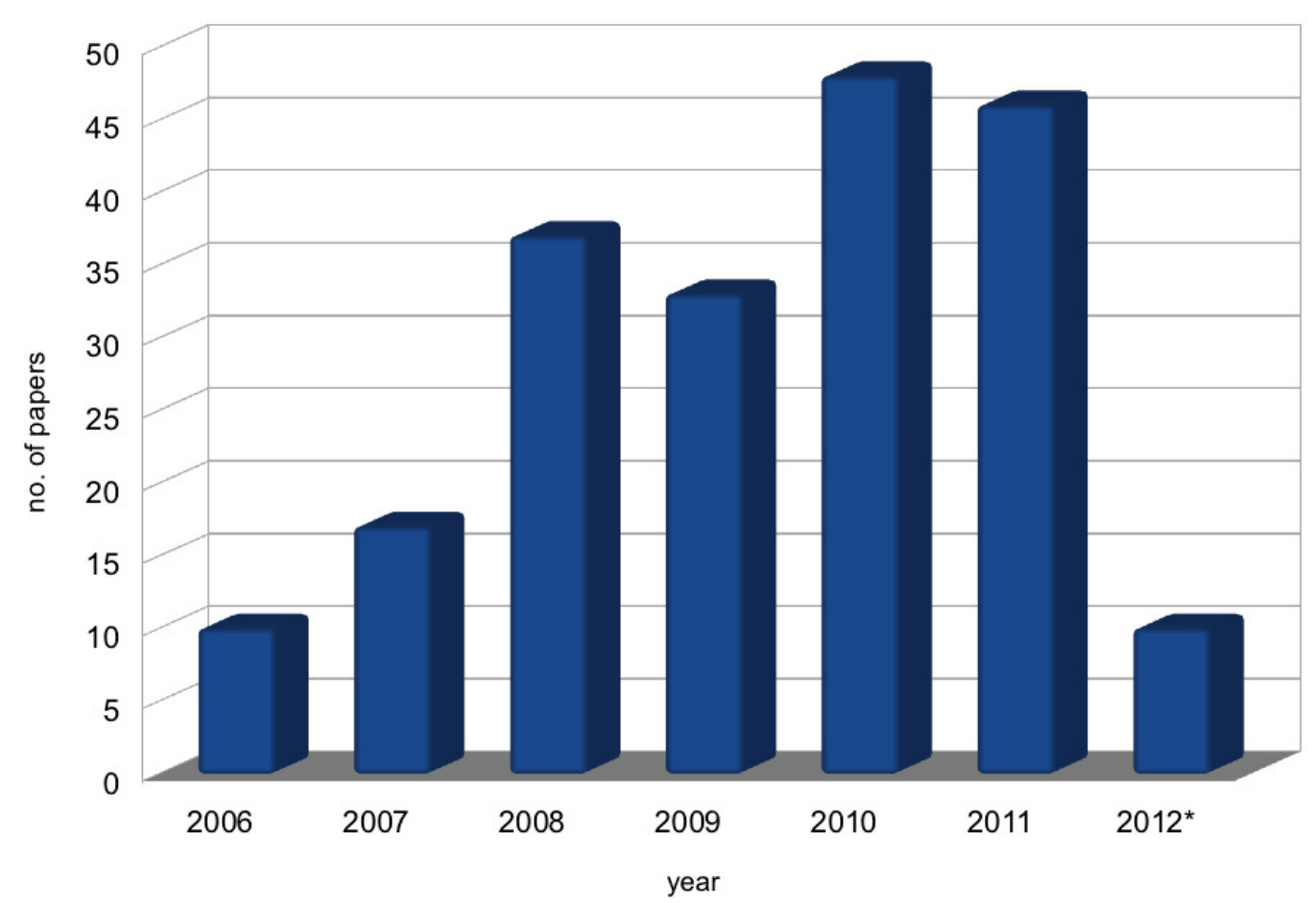

Figure 1: Number of papers addressing "greenhouse" and "wireless networking" by year (year 2012 includes papers indexed in Google Scholar from January through mid-June).

\section{Background and motivation}

Over several recent years a major part of computing technology had gone embedded and became connected. This shift was enabled by at least three important achievements of the semiconductor industry. First, the integration density and miniaturization allowed embedded devices to enjoy significant lifetimes on a single battery charge while still maintaining small volume and compact form factors. Second, the development of easy to use wireless communication interfaces relaxed the dependence of the connectivity on the underlying infrastructure together with batterypowered operation allowing for increased levels of autonomy. Finally, the technology has enabled the integration of all system's components onto a single integrated System on a Chip (SoC) platform featuring computational cores (microprocessors), volatile and non-volatile memories, wireless and wired interfaces, advanced power management, analog-to-digital interfaces, as well as more and more often integrated and miniaturized electronic or microelectro-mechanical (MEMS) sensors of various kinds. Among many other advantages - e.g., the reduction of development costs of complete systems - such advanced integration have succeeded in minimizing the sleep power consumption of these chips to the extent that has allowed to sustain a substantial autonomy to more and more sophisticated applications. Consequently, a whole spectrum of low to medium cost embedded battery-powered devices networked for all or most of its lifetime gave rise to ubiquitous computing - a growing volume of ambient networked computations starting to pervade our ecosystem [32] and serve as increasingly common commodity. A part of this spectrum is occupied by Wireless Sensor Networks (WSN) - connected systems of ultra low cost embedded computing nodes equipped with elaborate sensing capacity, some limited computational power, storage, and the ability to 
wirelessly communicate with each other. The term "network" is actually slightly misleading, because rather than providing sheer connectivity and data exchange, WSNs are more distributed systems resposible for collaboratively collecting spatio-temporal characteristics of physical variables. In fact, Delin [11] calls them measurement macro-instruments for their collaborative intelligence and a common set of objectives. As such WSNs find applications in many precise yet spatially distributed monitoring applications.

WSNs are distinguished from other types of networks of connected embedded devices by a handful of properties and characteristics. First of all, their design is primarily driven by low cost and low power consumption. This is because they are supposed to be ultra cheap and batterypowered in order to be deployable at large scale and not to need collecting after the deployment has finished its operation. They are also designed to be operated in infrastructureless environments, therefore all networking is provided by WSN nodes themselves and does not depend on any backbone infrastructure (e.g., as compared to cellular networks). This very feature implies multihop peer-to-peer communication to be the primary mechanism for providing through-network connectivity. A consequence of this is that low power requirement not only drives the construction of WSN nodes themselves (e.g. by involving relatively low computational power and short range small bandwidth communication), but more importantly predetermines network architectures and message passing (routing) protocols. All those components should be optimized for power dissipation and fairly uniform energy budget usage - both per node as well as network total.

Maximized energy efficiency of the network supports its second distinctive trait - an ability to operate in infrastructure-less, often remote, and sometimes even hostile environments with minimized, or even without human intervention [3]. This requires the network to have the ability to self-organize and re-organize independently of its scale, some number of node outages, or wireless links fragility. Relying heavily on redundancy in the network, this ability once achieved has an useful by-product - if the deployment environment is not hostile and the nodes' initial locations can later be manipulated by a human operator, incremental modifications are easy to perform because nodes are infrastructure independent and network will automatically re-adjust itself after such modifications.

The simplest way to use WSNs is just to let them act as hubs of "wireless extension coords" for the sensors passing through a network to some central base station measurements taken at the schedule orchestrated by a node's microprocessor. However, one of the most interesting application-specific optimization that can be performed in WSNs is data aggregation. It is based on an observation that WSNs are typically not data networks organized around transmitting all collected data to some central base station(s), they are more information networks responsible for providing preferably only useful information to the application layer, in many cases, ideally, only actionable information [7]. For instance, an event-driven sensor network will act only if the observed variable changes its value by some significant amount (greater than some minimum threshold) filtering out variations in measurements, thus reducing traffic as well as network's power dissipation [30]. Other application, like, e.g., frost sensor will not be transmitting temperature until it gets within a certain range from the freezing point [7]. In other words, nodes in sensor network can use their distributed computational intelligence to contain data transmission within local circles of nodes (clusters, or single nodes) and transmit globally only useful information rather than raw sensor data. Such behavior has a major impact on the communication intensity therefore on power consumption as well as network architecture. Consequently, since defining what higher-level information can be extracted from raw sensor data is application-specific, so should be WSN deployments.

Since their invention, WSNs have matured significantly as well as produced several long 
International Journal of Wireless \& Mobile Networks (IJWMN) Vol. 4, No. 4, August 2012

term or even permanent deployments [3]. That also includes agriculture, e.g., [4, 7, 20, 46]; extensively surveyed recently by $[36,43]$. As a consequence of this progress two prominent advantages have become clear: first, WSNs ability to provide detailed spatio-temporal characteristics of environments and processes broadens significantly the understanding of the underlying application. As a consequence, it enables sophisticated optimizations of the application. Second, although generic approaches and deployments are obviously possible, it is clear that WSNs and applications they are being applied to are heavily inter-dependent and efficient WSN deployments require application-specific customizations. Consequently it becomes increasingly important for domain specialists to understand WSNs and their implications on the application domain, as well as for computer engineers to understand applications and exploit peculiarities allowing WSN deployments to enjoy maximized performance and usability.

Modern greenhouse is one of such promising applications of WSNs that exhibit a lot of peculiarities that may make a tremendous impact on WSN deployments. In short, greenhouse is a crop cultivation structure fairly well isolated from the influence of the external environmental factors (often actually referred to as disturbances), therefore commonly operated as a closedloop control system. However all the major physical processes going on in the greenhouse expose gradients as well as spatial and temporal variability, i.e., they are perfect testbeds for evaluating WSN deployments. Particularly, because today's automation infrastructure operating within the greenhouse is usually wired, and as such poses some certain limitations to the granularity of the information about the processes inside the structure that can be collected in an economically justified fashion. This is because wires are costly to lay out and these costs may soar even up to $\$ 650$ per meter [36]. Wired infrastructure also requires ubiquitous supply power because wired transmissions over long distances requires a lot of energy to be dissipated [16]. Consequently, wired equipment is fairly immobile because changes in architecture of the system are difficult to conduct therefore, yet again, costly. Finally, wired infrastructure is also more difficult to scale because cabling and routing creates overheads in terms of complexity and cost that does not scale linearly with the number of terminal nodes in the network [31].

On the contrary, as discussed above, WSNs deliver their computational and communication infrastructure in the form of a collection of low-cost, self-organizing, battery-powered, autonomous nodes [22]. Their computational and radio communication capacities are dirt cheap - starting from \$1-\$2 per node - such costs are negligible when compared to the price of some of the sensors typically employed in the agriculture [21]. Autonomy and self-organization result in high mobility - the user can relocate nodes at will with very little concern about the complete system's operation. Even when a disconnectivity occures, additional nodes can be introduced incrementally to restore system's operation. Since, WSN nodes can provide both sensing and routing functionality, the problem of network's scaling becomes software-defined - it gets abstracted to the routing management software layer and as such becomes detached from the network's size. On the other end, WSNs have certain drawbacks and pose certain challenges as well. First of all, as mentioned before, their autonomous and low-power operation is mainly traded off for lower computational power, storage space, communication bandwidth and communicable range available to the node. Moreover, the radio link is much less reliable than the cable link as well as much less secure [22]. These constraints can either limit applicability or counter the benefits of WSN application. E.g., implementing security or reliability measures can increase power consumption and as a consequence limit the much desired autonomy.

Despite of all those concerns, greenhouse management is one of applications where WSNs may actually make an impact on the complete production ecosystem's economics. Fairly well understood, crop growth is actually not controlled nowadays in a very fine grained fashion, e.g., 
International Journal of Wireless \& Mobile Networks (IJWMN) Vol. 4, No. 4, August 2012

many papers report that only a single wired sensor is usually responsible for observing greenhouse's climate state. Consequently, greenhouse control systems could actually directly utilize more detailed knowledge of the crop growth process and result in almost instantaneous increase in both yield and productivity. However greenhouses are not one application, they vary size and architecture, geographical location, type of crops being grown, many more [41, 42]. All these factors may influence both the performance and the architecture (therefore cost) of the network. Consequently, from the perspective of installing and operating WSNs inside them, greenhouses constitute a broad spectrum of design points, each of posing different challenges. For each installation many feasible solutions may actually exist, but optimizing performance/price factor of the deployment may require a significant amount of insight.

On the other hand, while the body of work on deploying WSNs in greenhouses is growing quickly, few of those works focus on anything more than just describing an operable deployment of the network, and/or the architecture of the node(s) constructed for the particular installation. Few works, do actually make an effort to characterize their proposals in terms of such basic metrics as deployment cost [21], or the estimated lifetime of the node [24, 50], not to mention that of a complete deployment. To the best of our knowledge, no paper deals with reliability of the communication nor with its security, while those are quite obviously serious issues in the greenhouse with reliability being specifically application-specific as both climate control as well as crop development may be perpetually impacting the communication range and radio wave penetration, therefore the link power budget. Finally, there is a severe lack of comparative studies of the performance of different WSN technologies and architectures within the greenhouse application domain. Apart from the domain being quite immature, we identify the reasons for this state as being twofold: first, the horticulture researchers do not have enough understanding of the design space of wireless communication technology, second, the computer and communication researchers have little understanding of the underlying application domain. As a consequence of this conjecture, the purpose of this work is to contribute to closing this gap in understanding. As such, this paper does not attempt to be a survey reviewing each, or most of the research in the domain. Such reviews were recently included in more general surveys on WSN applications in agriculture [43, 36]. Nor its the survey of WSN state-of-the-art technologies and available devices, as such more or less thorough surveys are a part of many papers in the domain, e.g., [15, 24, 25]. Rather than that, based on some preliminary discussion and field work of ours, we will make an attempt to survey the problem's design space, and make some observations about the possible directions and approaches to solutions.

\section{Greenhouses for Computer Engineers}

Greenhouse is a structure that attempts at creating an optimized environment for crop growth. It is being done primarily by isolating the cultivation process from the adverse influence of external environmental factors. A direct consequence of this isolation is the possibility of controlling the greenhouse inside climate parameters both manually and automatically. Nowadays, greenhouses are one of the forefront examples of the so called precision agriculture - an approach to optimizing the agricultural production process by means of ubiquitous monitoring and sophisticated control of the crop growth applied at increasingly fine-grained resolution in order to fulfill the structure's primary objective: maximize yield while minimizing costs and environmental impact of the production process [6].

Crop growth is primarily influenced by the surrounding climate, as well as the amount of water and fertilizers supplied by the irrigation system [8]. Therefore in a typical industrial setup, the problem of optimizing crop growth is reduced to the problem of controlling the state of the greenhouse's climate and its irrigation and fertilizers' supply (fertirrigation) in a manner that allows to align its state with the requirements of a specific type of crops. Defined as such, the 
International Journal of Wireless \& Mobile Networks (IJWMN) Vol. 4, No. 4, August 2012

process is essentially decomposable into two independent components: the climate control and the fertirrigation control, both allowing for the closed-loop automatic control [8]. Depending on the geographical location, one of those systems tends to be more important than the other: water management is going to be more important in desertous regions with restricted water supply, while climate management is going to be more important in colder regions with limited periods of vegetation.

Some recent papers actually observe that the objective of maximizing crop growth gives an incentive to monitor the crop itself and factor these observations in into the control loop. Examples include monitoring leaves humidity and temperature as indicators of crop diseases [8]. Extrapolating from such a trend, we can expect that the granularity of monitoring may go even below the resolution of a single plant in the future. However, the infrastructure required needs to be economically justified by the yield increase.

\subsection{Climate control}

The growth of the plants is the result of the photosynthesis process which converts water and and carbon dioxide into glucose and oxygen [1]. Plants are sensitive to the the portion of light spectrum from 400 to $700 \mathrm{~nm}$ wavelength, or so-called photosynthetically active radiation [39]. Photosynthesis process occurs during daytime, when plants are active. The speed of glucose production depends on the proper temperature levels, therefore temperature control dominates the climate control in the greenhouse. The climate inside a greenhouse is defined by two dominant physical processes though: energy transfer - both heat and radiation, and mass balance - including both water vapour fluxes and $\mathrm{CO}$ concentration [8]. In order to manipulate these processes, physical variables related to them have to be observed other controlled. For climate control in the greenhouse the observables include most importantly temperature, humidity, light (solar radiation) and CO concentration. Also soil (substrate) temperature and moisture impacts the internal climate and requires monitoring. Finally, a handful of external factors disturb the internal climate: external temperature and humidity, wind speed and direction, as well as solar radiation [5]. Once measured, the climate's internal state can be augmented by injecting or removing heat (though heating and ventilation), moisture (sprinkling or fogging and ventilation), and light and radiation (shades and artificial lighting).

A direct consequence of the fact that the photosynthesis process occurs during the day is that the inside temperature of the greenhouse has two set points or states: diurnal, optimized for plant growth, and nocturnal, when the temperature is reduced to safe levels while allowing to minimize the resource utilization. The second most important parameter of the inside climate of the greenhouse is humidity. High humidity may increase probability of diseases and reduce transpiration. Low humidity causes hydria stress, causing the stomata to close and decreasing the speed of the photosynthesis process. On the other hand, humidity impacts temperature as water vapours alter heat conductivity. Therefore temperature and humidity are inter-dependent and to some extent controlled by the same set of actuators, with the temperature control being of the top priority because of its impact on crop development.

\subsection{Water and fertilizers management}

Water is one of the two basic components of the photosynthesis equation, therefore one of the most important commodities in the system. Accurate irrigation is capable of delivering water necessary to the photosynthesis process, reduce crop susceptibility to diseases, and maximize crop growth though proper crop fertilization. Finally, precise water management minimizes the environmental impact of the production process. Like with moisture, under-irrigated crops are subject to hydria stress which result in production losses, while over-irrigation leads to plants' exposure to diseases as well as nutrient leaching [18]. As a carrier of fertilizers water provides transport for the soil or substrate conditioning which is essential to optimal crop growth [8]. 
International Journal of Wireless \& Mobile Networks (IJWMN) Vol. 4, No. 4, August 2012

Precise irrigation have been reported to reduce water usage even by up to $60 \%$.

The problem of water management should be relatively easy as modern irrigation systems can apply water uniformly even over large areas in an open loop control. Principally, such an irrigation system decides on [17]:

- how long the irrigation session should last,

- how often the irrigation sessions should be repeated; and

- how much water and/or fertilizers should be used.

However, spatial as well as temporal variations in soil condition create an opportunity to optimize both irrigation and fertilization using closed-loop control. For that purpose variablerate irrigation systems require spatially and temporally distributed measurements of the soil condition to be available [19]. The specific set-up point of the irrigation system is partially determined by some global parameters like the kind of crops being grown, the type of soil, leaf coverage, the stage of growing. Other key variables in soil condition monitoring have to be measured. That set typically includes temperature and/or heat radiation of the soil, moisture content, acidity levels ( $\mathrm{pH}$ value), salinity, and electric conductivity.

In greenhouses and nurseries, the variance in soil conditions as well as the efficiency of fertirrigation is often optimized by utilizing artificial substrates with desirable physical parameters such as total pore space, air-filled pore space, water-holding capacity, bulk density, wettability and drying rates; as well as chemical properties like $\mathrm{pH}$, electrical conductivity and mineral nutrient content [12]. Such substrates reduce variance in susbtrate parameters but not necessarily eliminate it, due to spacial and temporal gradients of other climatic variables of the greenhouse that influence the substrate state. Also the uniform conditioning of the soil does not necessarily translate into the uniform growth of the plants therefore precise crop monitoring and closed-loop irrigation may still be beneficial.

Finally, soil condition correlates to the inside climate - particularly soil temperature as well as its moisture content are interrelated with the inside climate state of the greenhouse. therefore the water management and climate control are not completely independent systems.

\subsection{Production management}

If a greenhouse is taken as an end-to-end system, several other factors may require monitoring and control. Crop cultivation is both manual labor and asset intensive, consequently the usage of both labor and assets can be a subject to optimization [27]. The economical objective here is to increase the end-to-end efficiency, and consequently reduce the overall crop production costs. For that purpose, tools and machinery tracking may be introduced and based on the collected information the location of the storage areas for tools and machinery, routes and procedures can be optimized to maximize accessibility [45]. Moreover, human workers can also be tracked (both passively and actively, e.g. with the help of check-in kiosks) in order to inform the management system about their activity. This may be particularly useful for labor optimization in greenhouses operated in North America or Nothern Europe, where workers are typically foreign citizens and may be experiencing a severe language barrier.

For tracking and identification radio frequency technologies other than WSNs tend to be more suitable, although the use of WSNs is also possible. Primarily, the radio frequency identification (RFID) technology is actually designed for tracking goods and has found many uses in agriculture [35]. However, its utilization in the greenhouse, so far is at its infancy and mostly overlaps with applications of WSNs, i.e., remote sensing, e.g., monitoring climate. So far however, almost no research is available touching on RFID-based tracking and location monitoring benefits to greenhouse management and we mostly interpolate results coming from similar domains [45]. 
International Journal of Wireless \& Mobile Networks (IJWMN) Vol. 4, No. 4, August 2012

RFIDs for tracking have found its use in nurseries, where each plant grows in the individual pot. Pots can be tagged by burying an RFID tag inside the pot and then plants can be tracked individually at a very fine-grained resolution. Definitely, in the greenhouse there is a lot of potential for tracking different objects related to crop transfer, tools and machinery movements, as well as human workers activity. With localization though there is a difficulty because changing crop green mass volume tends to change the propagation of the radio wave inside the greenhouse therefore localization based on radio signal strength characterization may be problematic or require challenging calibration.

Summarizing, not only the climate and irrigation systems can be optimized using distributed wireless sensing to maximize the yield of the greenhouse, but also the process of cultivation and harvesting can be improved in terms of its labor and assets intensity. This requires enough detailed information to be extracted and abstracted by tracking and monitoring locations of items and workers, then constructing models in order to produce actionable information. Considering high and perpetually rising labor as well as tools and machinery costs, it is surprising how little research interest has been dedicated so far to this problem.

\section{Wireless Sensor Networks in Greenhousse}

There are certain specific aspects of utilizing WSNs in greenhouses that are almost left unaddressed in the current body of literature on the subject. Some simple and easy to agree upon observations were mentioned in the discussion above, nevertheless some require more elaborate argumentation that will be given in this section.

\subsection{In Greenhouse Radio Communication}

As mentioned before, greenhouses are not uniform structures [42] and as such do not provide uniform conditions for the radio signal propagation. One of the low-level decisions that a designer of a WSN has to make is what ISM band his network is going to operate within. This decision has certain consequences in the greenhouse application. The typical $2.4 \mathrm{GHz}$ bandwidth has standardized WSN solutions like IEEE 802.15.4 physical and media access layer definition, together with a common industry standard ZigBee architecture running over the top of 802.15.4. The advantages of using ZigBee are quite obvious. Standardized protocol stack and easy to use at the application layer isolates the designer from the low level network issues. Self-organization of the network, routing algorithms and other aspects of the through-network communication are encapsulated in the ZigBee stack [48]. The fact that $2.4 \mathrm{GHz}$ band is usually considered crowded and interference-prone should not have much importance in a typical greenhouse. Power consumption of a ZigBee device is low and depending on the generation and the specific parameters of the radio chip should typically be in range of $20 \mathrm{~mA}$ to $30 \mathrm{~mA}$. Finally the cost of a ZigBee chip is low, again depending on configuration from $\$ 1$ to $\$ 3$. Consequently many researchers used ZigBee as their communication infrastructure and reported successful operation of the network $[13,28,29,37,38,40,44,47,48,49,50,51]$. The communication range reported varies from $5 \mathrm{~m}$ to approx. $30-70 \mathrm{~m}$ depending on the author [14].

The problem with ZigBee and other $2.4 \mathrm{GHz}$ solutions is however related to the change in conditions inside the greenhouse during the crop growth period. If the type of crop being grown is tall and produces a large volume of a green mass, the communication range using ZigBee technology may not only dramatically reduce, it will also vary by orders of magnitude within the crop growth cycle. Our measurements show that the initial range of a few dozen meters may drop down as drastically as two orders of magnitude, to 1-2 meters in case of tall crops like for instance paprika [15]. If so, a large greenhouse of say $200 \mathrm{~m}$ length and $100 \mathrm{~m}$ width would require approx. 5000 nodes to provide worst-case connectivity, which is a very complex network in fact beyond ZigBee addressing capacity. Most wastefully, this worst-case would constitute an over-design for a considerable chunk of network's lifetime. 
Since sensor nodes do not have to be ultra miniaturized for the greenhouse application, other options also quite extensively used in the literature are the so-called sub-1GHz ISM bands namely a global band of $433 \mathrm{MHz}$ and the regional bands like $900 \mathrm{MHz} 3$. Although they are also susceptibile to green mass volume change, but according to our measurements the worst case of using a $+10 \mathrm{dBm}$ transceiver at $433 \mathrm{MHz}$ band is about $25 \mathrm{~m}$ while at $900 \mathrm{MHz}$ it is about a half of this range. Consequently the same size hypothetical greenhouse can be fully connected with approx. 32 nodes $(433 \mathrm{MHz})$ or 140 nodes $(900 \mathrm{MHz})$ which is even two orders of magnitude drop in network's scale as compared to ZigBee. The disadvantage of these bands are increased quad pole antenna sizes (up to $17.5 \mathrm{~cm}$ for $433 \mathrm{MHz}$ band) but the application such as greenhouse can actually sustain such a requirement. Note however, that changing the ISM band does not solve the network's worst-case over-design, as open field communication at $433 \mathrm{MHz}$ and $900 \mathrm{MHz}$ reaches a range of about $2 \mathrm{~km}$ and $1 \mathrm{~km}$, respectively using a $+10 \mathrm{dBm}$ transceiver.

The sub-1GHz ISM bands have also another advantage. The penetration of the sub- $1 \mathrm{GHz}$ waves is much improved over $2.4 \mathrm{GHz}$ and as a result the coverage is much more uniform around the node located inside the structure. Yet still one of the architectural challenges of using WSNs in greenhouses barely considered in up-to-date literature is the fact that the organization of the greenhouse creates open spaces which work like long-range "channels" for the radio signal. In other words, the $433 \mathrm{MHz}$ through-crops range of say $25 \mathrm{~m}$, gets extended to a few hundred meters (effectively end-to-end even in a large scale greenhouse), if nodes are located over the crops level or on the verge of the plant rows.

A few important research implications emerge from these observations. First, as plants grow, the worst-case conditions for the wireless communication change and in extreme cases may reduce or increase by almost two orders of magnitude during the growth cycle. Consequently, what was an optimized setup of a WSN at one stage of its lifetime, may experience disconnects and/or interferences at some other stage. The differences may be so huge that they must be dynamically mitigated by the network by means of adjusting transmission power levels dynamically during the crop growth cycle. The development of specific algorithms to adjust power level dynamically, as well as application-specific configurations to minimize their overheads is a chunk of fundamental research work to be investigated in the future. Importantly, it is quite remarkably greenhouse specific problem as not many other applications experience such dramatic variations in penetration and range of the radio signal. A second important implication of the radio interface behavior is architectural, and unfolds as follows. From the discussion above it becomes clear that the a proper decomposition of the network and location of the nodes may change the network complexity and scale significantly. Putting infrastructure nodes (access points, routers) over the crop level may change the network scale estimations and provide long-range connectivity between fairly isolated clusters of in-the-crops nodes. It becomes clear, that there is another piece of fundamental research to be conducted to resolve this issue and propose optimized deployment schemes. Finally, it is quite clear, that it would be beneficial before going to applications, to perform a four dimensional (length, width, height, time) characterization of transmission quality over different ISM bands as well as construct accurate simulation models of wireless communication in the greenhouse in order to facilitate rapid architectural explorations. While simulation models for the greenhouse exist [30], they are focused on simulations of the application not of the radio communication quality. Without a detailed insight into the RF signal propagation being a result of such characterization it is going to be extremely difficult to deploy optimized networks that monitor greenhouse processes at the resolution of a single plants according to the currently observable trends.

3 868 MHz in Europe and 915MHz in the USA 
International Journal of Wireless \& Mobile Networks (IJWMN) Vol. 4, No. 4, August 2012

\subsection{Greenhouse WSN Architecture}

Another issue barely touched by the literature so far is the impact of the distribution of sensing tasks across the WSN on the overall deployment cost. As mentioned before, computation and communication capabilities of a sensor node contribute to a minute cost of the node, and most of the Bill of Manufacturing (BoM) is consumed by the costs of the sensors. If so, in real commercial deployments, the actual assignment of the specific sensing tasks to nodes will have a tremendous impact on the deployment cost. Consequently research is needed on network architectures where different nodes have different sensing functions in a fashion similar to that of [25]. Exploratory research should be performed in synchronization with horticultural researchers to provide insight into the decomposition strategies of sensing tasks that need to be done, as well as their distribution across the network architecture. As costs and price/performance ratios of the deployment are going to be a primary factors for the growers considering WSN deployments in their greenhouses their incentives need to be solidly characterized by the research community.

Another application-specific architectural issue is that typically WSNs are designed and/or researched as systems that are being deployed at random locations, self organize and assure connectivity through redundancy [33]. While this is a feasible solution in the greenhouse, typically the use case is different: the grower precisely knows what locations of the sensor and actuator nodes he wants to use, what he does not know and likely has no expertise in is the deployment of the infrastructure nodes: access points and routers. On the other hand, the variance in radio propagation that is observed inside the greenhouse makes the problem of optimized deployment of such infrastructure nodes not trivial, especially if large deployments are to be considered. Therefore the research needs to be conducted on the deployment optimization that takes into consideration peculiarities of the greenhouse application. It is important to note though, that the infrastructure nodes are not supposed to be expensive because they are not likely to carry expensive sensors on them, therefore the problem of redundancy is not so important from the network cost point of view. However, redundancy may cause interference, therefore reduce end-to-end reliability of the transmissions which consequently requires countermesures that reduce lifetime and bandwidth.

\subsection{Reliability and Security of Communication}

Reliability requirements for wireless communication within the greenhouse is another majorly abandoned topic in the body of related literature. Researchers reporting successful deployments cite approximate packet reception rates from $60 \%$ to $100 \%$. While the physical processes in the greenhouse are not changing very rapidly and such variance is acceptable for monitoring applications, control applications require strict quality of service guaranties. For instance, it is not acceptable, or may get extremely costly, that the control system opens the irrigation valve that will not have been closed due to wireless link fragility or sensor node outage. Consequently reliability has to be factored in when discussing WSN deployments in the greenhouse. Fortunately, the problems here do not vary significantly from the general state-of-the art and the body of literature exits on WSN reliability including some methods of providing end to end reliability, e.g., [2]. The research on WSN reliability has recently been surveyed in [9]

Also security is always of a great concern when moving sensitive transmissions from wired channels to wireless. Although greenhouses are typically detached and enclosed areas their information networks carry sensitive information that may cause significant losses and damage if breached. Therefore cryptografic security of most transmissions is a must. Pretty much the only research question here is whether the information spectrum inside the greenhouse can be graded according to sensitivity and secured at different security levels. The reasons for doing so is an obvious trade-off between cryptografic security of transmissions and the network's lifetime. Also denial-of-service detection and prevention has to be considered if the presence of 
International Journal of Wireless \& Mobile Networks (IJWMN) Vol. 4, No. 4, August 2012

malicious transmissions cannot be excluded. Again, the techniques here are pretty much standard to WSNs although specific configurations may be application dependent [23].

\subsection{Energy Harvesting}

While the autonomy reported in many papers vary from approx. 160 days to more than a year, these results does not seem to sustain a scalable network of say 5000 nodes and beyond. A quick mental experiment illustrates that in a continuous operation of a network of 5000 or more, 160 days of autonomy gives approximately 32 nodes a day that need battery replacement. This may become a serious hassle when a network of such or greater scale enters the production environment. Unlike in many other environments, greenhouse's climate is artificially controlled and a lot of energy is being both injected and removed from the structure all of the time. Consequently energy harvesting seems to be a natural solution to create a zero-net-energy sensor nodes to which power is delivered not by means of power distribution network, but by light, heat, vibrations and other sources of ambient energy being injected anyway into the structure. Particularly interesting is obviously light, as it is ubiquitous in the greenhouse and reasonably easy to harvest [14]. Other obvious sources include power generation from the temperature difference as powering WSN nodes from a minute difference in temperatures was already commercially demonstrated [26], and temperature states in the greenhouse are actually most carefully controlled and monitored, therefore could be budgeted quite accurately. Other possible sources include electromagnetic [10], vibration, and pervading RF signals [34]. A solid characterization of the actual presence of the ambient energy sources inside the greenhouse vs. costs of their actual harvesting requires research focus in order to obtain application-specific cost/performance optimized commercially viable solutions. One of the ideas here may be to replace a global power distribution network with a distributed system of local integrated harvesting stations for the purpose of more energy-hungry nodes, while providing a lightweight individual harvesters for less demanding nodes. Such harvesting stations could for instance attempt at extracting air flow energy from the ventilation system.

\section{Conclusion}

In this paper, we reviewed the landscape of the application of Wireless Sensor Networking in greenhouse management. We surveyed the essentials of greenhouse operations so the computer engineer may undestand the basic complexities and the design space of the system and the need for Wireless Sensor Networking applications; next we briefly surveyed Wireless Sensor Network technology so the horticultural engineer can understand the not only the primary properties of WSNs, but also its design space and the approaches to optimizing its cost/performance ratio. Finally, we identified, formulated and discussed the design space of a few in our opinion most important problems in developing efficient and cost effective WSN deployments for greenhouses. We also put some stress on the observation that there is a justified need of system level design that cross-exploits the specifics of greenhouse management and the capabilities of modern WSN technology. The reasons to propose such an analysis have come from the gap we have observed between the results and observations coming from our preliminary field experimentation in the greenhouse, and the level of insight that the vast majority of research papers in the field are dealing with. 
International Journal of Wireless \& Mobile Networks (IJWMN) Vol. 4, No. 4, August 2012

\section{Acknowledgements}

The author would like to thank Mr. Nick Ray of Priva B.V., NL for providing access to the operating greenhouse, as well as for at least 12 months of fruitful and vision-sharing discussions.

This publication has been prepared as part of the project of the City of Wroclaw, entitled "Green Transfer" - academia-to-business knowledge transfer project co-financed by the European Union under the European Social Fund, under the Operational Programme Human Capital (OP HC): sub-measure 8.2.1.

\section{References}

[1] T. Ahonen, R. Virrankoski, and M. Elmusrati. Greenhouse monitoring with wireless sensor network. In 2008 IEEE/ASME International Conference on Mechtronic and Embedded Systems and Applications, pages 403-408. IEEE, Oct. 2008.

[2] Ö. Akan and I. Akyildiz. Event-to-sink reliable transport in wireless sensor networks. IEEE/ACM Transactions on Networking (TON), 13(5):1003-1016, 2005.

[3] I. Akyildiz. Wireless sensor networks: a survey. Computer Networks, 38(4):393-422, Mar. 2002.

[4] R. Beckwith, D. Teibel, and P. Bowen. Report from the field: results from an agricultural wireless sensor network. In 29th Annual IEEE International Conference on Local Computer Networks, pages 471-478. IEEE (Comput. Soc.), 2004.

[5] N. Bennis, J. Duplaix, G. Enéa, M. Haloua, and H. Youlal. Greenhouse climate modelling and robust control. Computers and electronics in agriculture, 61(2):96-107, 2008.

[6] S. Blackmore. Precision farming: an introduction. Outlook on agriculture, 23(4):275-280, 1994.

[7] J. Burrell, T. Brooke, and R. Beckwith. Sensor and actuator networks - vineyard computing: sensor networks in agricultural production. IEEE Pervasive Computing, 3(1):38-45, Jan. 2004.

[8] D. Chaudhary, S. Nayse, and L. Waghmare. Application of wireless sensor networks for greenhouse parameter control in precision agriculture. International Journal of Wireless \& Mobile Networks (IJWMN) Vol, 3(1):140-149, 2011.

[9] D. Chen and P. Varshney. Qos support in wireless sensor networks: A survey. In International Conference on Wireless Networks, volume 233, 2004.

[10] W. Chye, Z. Dahari, O. Sidek, and M. Miskam. Electromagnetic micro power generator: A comprehensive survey. In Industrial Electronics Applications (ISIEA), 2010 IEEE Symposium on, pages $376-382$, oct. 2010 .

[11] K. A. Delin. The sensor web: A macro-instrument for coordinated sensing. Sensors, 2(7):270-285, July 2002.

[12] M. Evans. Greenhouse and nursery growing substrates. Accessed online on June 2012: http://bumperscollege.uark.edu/hort/5493.php, 2012.

[13] G. He, X. Wang, and G. Sun. Design of a greenhouse humiture monitoring system based on zigbee wireless sensor networks. In 2010 Fifth International Conference on Frontier of Computer Science and Technology, pages 361-365. IEEE, Aug. 2010.

[14] J. Hou and Y. Gao. Greenhouse wireless sensor network monitoring system design based on solar energy. In 2010 International Conference on Challenges in Environmental Science and Computer Engineering, volume 2, pages 475-479. IEEE, 2010.

[15] J. Hwang, C. Shin, and H. Yoe. A wireless sensor network-based ubiquitous paprika growth management system. Sensors, 10(12):11566-11589, Jan. 2010.

[16] M. Ilyias and I. Mahgoub, editors. Handbook of Sensor Networks: Compact Wireless and Wired Sensing Systems, volume 4. CRC Press, 2005. 
International Journal of Wireless \& Mobile Networks (IJWMN) Vol. 4, No. 4, August 2012

[17]P. Kia, A. Far, M. Omid, R. Alimardani, L. Naderloo, et al. Intelligent control based fuzzy logic for automation of greenhouse irrigation system and evaluation in relation to conventional systems. World Applied Sciences Journal, 6(1):16-23, 2009.

[18] Y. Kim and R. Evans. Software design for wireless sensor-based site-specific irrigation. Computers and Electronics in Agriculture, 66(2):159-165, 2009.

[19] Y. Kim, R. Evans, and W. Iversen. Remote sensing and control of an irrigation system using a distributed wireless sensor network. Instrumentation and Measurement, IEEE Transactions on, 57(7):1379 -1387, July 2008.

[20] K. Langendoen, A. Baggio, and O. Visser. Murphy loves potatoes: experiences from a pilot sensor network deployment in precision agriculture. In Proceedings 20th IEEE International Parallel \& Distributed Processing Symposium, page 8 pp. IEEE, 2006.

[21] J. Lea-Cox, A. Ristvey, F. Arguedas Rodriguez, D. Ross, J. Anhalt, and G. Kantor. A low-cost multihop wireless sensor network, enabling real-time management of environmental data for the greenhouse and nursery industry. In International Symposium on High Technology for Greenhouse System Management: Greensys2007 801, pages 523-530, 2007.

[22]F. L. Lewis. Wireless Sensor Networks, pages 11-46. John Wiley \& Sons, Inc., 2005.

[23] J. Lopez. Wireless sensor network security, volume 1. Ios Pr Inc, 2008.

[24] J. A. López, F. Soto, P. SÃ̄inchez, A. Iborra, J. Suardiaz, and J. A. Vera. Development of a sensor node for precision horticulture. Sensors, 9(5):3240-3255, 2009.

[25] J. López Riquelme, F. Soto, J. Suardíaz, P. Sánchez, A. Iborra, and J. Vera. Wireless sensor networks for precision horticulture in southern spain. Computers and Electronics in Agriculture, 68(1):25-35, Aug. 2009.

[26] Micropelt GmbH. Te-power node evaluation kit. Accessed online on June 2012: http://www.micropelt.com/applications/te_power_node.php, 2012.

[27] V. L. Narasimhan, A. A. Arvind, and K. Bever. Greenhouse asset management using wireless sensor-actor networks. In International Conference on Mobile Ubiquitous Computing, Systems, Services and Technologies (UBICOMM'07), pages 9-14. IEEE, Nov. 2007.

[28] N. Pang. Zigbee mesh network for greenhouse monitoring. In 2011 International Conference on Mechatronic Science, Electric Engineering and Computer (MEC), pages 266-269. IEEE, Aug. 2011.

[29]C. Park, H. Cho, D. Park, Y. Lee, S. Cho, and J. Park. Aoa localization system design and implementation based on zigbee for applying greenhouse. In 2010 th International Conference on Embedded and Multimedia Computing, pages 1-4. IEEE, Aug. 2010.

[30] A. Pawlowski, J. L. Guzman, F. Rodríguez, M. Berenguel, J. Sánchez, and S. Dormido. Simulation of greenhouse climate monitoring and control with wireless sensor network and event-based control. Sensors, 9(1):232-252, Jan. 2009.

[31] M. Pióro and D. Medhi. Routing, flow, and capacity design in communication and computer networks. Elsevier/Morgan Kaufmann, 2004.

[32] S. Poślad. Ubiquitous Computing: Smart Devices, Environments and Interactions. Wiley Online Library, 2009.

[33]D. Puccinelli and M. Haenggi. Wireless sensor networks: applications and challenges of ubiquitous sensing. IEEE Circuits and Systems Magazine, 5(3):19-31, 2005.

[34]M. Raju and M. Grazier. Energy harvesting. ulp meets energy harvesting: A game-changing combination for design engineers. Accessed online: http://www.ti.com/lit/wp/slyy018a/slyy018a.pdf, 2010.

[35]L. Ruiz-Garcia and L. Lunadei. The role of rfid in agriculture: Applications, limitations and challenges. Computers and Electronics in Agriculture, 79(1):42 - 50, 2011. 
International Journal of Wireless \& Mobile Networks (IJWMN) Vol. 4, No. 4, August 2012

[36]L. Ruiz-Garcia, L. Lunadei, P. Barreiro, and I. Robla. A review of wireless sensor technologies and applications in agriculture and food industry: State of the art and current trends. Sensors, 9(6):4728-4750, June 2009.

[37] J. Song. Greenhouse monitoring and control system based on zigbee wireless senor network. In 2010 International Conference on Electrical and Control Engineering, pages 2785-2788. IEEE, June 2010.

[38] B. Su and Y. Han. Design of greenhouse wireless monitoring system based on zigbee. In 2010 International Conference on E-Product E-Service and E-Entertainment, pages 1-4. IEEE, Nov. 2010.

[39]L. Tik, C. Khuan, and S. Palaniappan. Monitoring of an aeroponic greenhouse with a sensor network. International Journal of Computer Science and Network Security, 40(3):240-246, 2009.

[40] P. Tirelli, N. A. Borghese, F. Pedersini, G. Galassi, and R. Oberti. Automatic monitoring of pest insects traps by zigbee-based wireless networking of image sensors. In 2011 IEEE International Instrumentation and Measurement Technology Conference, pages 1-5. IEEE, May 2011.

[41]B. von Elsner, D. Briassoulis, D. Waaijenberg, A. Mistriotis, C. von Zabeltitz, J. Gratraud, G. Russo, and R. Suay-Cortes. Review of structural and functional characteristics of greenhouses in European Union countries: Part I, design requirements. Journal of Agricultural Engineering Research, 75(1):1 - 16, 2000.

[42] B. von Elsner, D. Briassoulis, D. Waaijenberg, A. Mistriotis, C. von Zabeltitz, J. Gratraud, G. Russo, and R. Suay-Cortes. Review of structural and functional characteristics of greenhouses in European Union countries: Part II: Typical designs. Journal of Agricultural Engineering Research, 75(2): $111-126,2000$.

[43] N. Wang, N. Zhang, and M. Wang. Wireless sensors in agriculture and food industry - recent development and future perspective. Computers and Electronics in Agriculture, 50(1):1-14, Jan. 2006.

[44] W. Wang and S. Cao. Application research on remote intelligent monitoring system of greenhouse based on zigbee wsn. In 2009 2nd International Congress on Image and Signal Processing, pages 1-5. IEEE, Oct. 2009.

[45] T. Wark, P. Corke, P. Sikka, L. Klingbeil, Y. Guo, C. Crossman, P. Valencia, D. Swain, and G. Bishop-Hurley. Transforming agriculture through pervasive wireless sensor networks. IEEE Pervasive Computing, 6(2):50-57, Apr. 2007.

[46] Y. Xiaoxue. Design and implementation of intelligent urban irrigation system ZigBee modules for sensor networking. In 2011 IEEE 2nd International Conference on Software Engineering and Service Science, pages 461-464. IEEE, July 2011.

[47]C. Yu, Y. Cui, L. Zhang, and S. Yang. Zigbee wireless sensor network in environmental monitoring applications. In 2009 5th International Conference on Wireless Communications, Networking and Mobile Computing, pages 1-5. IEEE, Sept. 2009.

[48]Q. Zhang, X.-1. Yang, Y.-m. Zhou, L.-r. Wang, and X.-s. Guo. A wireless solution for greenhouse monitoring and control system based on zigbee technology. Journal of Zhejiang University SCIENCE A, 8(10):1584-1587, Oct. 2007.

[49]Z. Zhiwei. Design of greenhouse monitoring system based on zigbee. In 2011 2nd International Conference on Artificial Intelligence, Management Science and Electronic Commerce (AIMSEC), pages 3895-3897. IEEE, Aug. 2011.

[50] Y. Zhou, X. Yang, X. Guo, M. Zhou, and L. Wang. A design of greenhouse monitoring \& control system based on ZigBee wireless sensor network. In 2007 International Conference on Wireless Communications, Networking and Mobile Computing, pages 2563-2567. IEEE, Sept. 2007.

[51] Y. W. Zhu, X. X. Zhong, and J. F. Shi. The design of wireless sensor network system based on zigbee technology for greenhouse. Journal of Physics: Conference Series, 48(1):1195-1199, Oct. 2006. 